\section{CPC-060 FOLLOW-UP OF PATIENTS TREATED BY PROLONGED- RELEASE OLANZAPINE IN A PSYCHIATRIC HOSPITAL}

doi:10.1136/ejhpharm-2013-000276.517

${ }^{1} \mathrm{~A}$ Aldequer, 'C Peloso, 'C Moutonnier, 20 Canceil, $2 \mathrm{~B}$ Garnier, $2 \mathrm{MN}$ Vacheron, ${ }^{1}$ E Advenier-lakovlev. ${ }^{1}$ Centre Hospitalier Sainte-Anne, Pharmacy, Paris, France; ${ }^{2}$ Centre Hospitalier Sainte-Anne, Psychiatry, Paris, France

Background Olanzapine is an atypical antipsychotic. Available in France since 2010, olanzapine pamoate (OP) is a prolonged-release suspension for intramuscular (IM) injection. OP is effective in the treatment of schizophrenic patients previously stabilised by oral olanzapine, and has been developed to improve compliance in these patients. In France, the injection must be performed in a psychiatric hospital department with 3-hour monitoring due to the potential 'post-injection syndrome' associated with OP.

Purpose To review the use and safety of OP since it became available in our hospital in May 2011.

Materials and Methods Retrospective study conducted from June 2011 to October 2012 in our 750-bed psychiatric hospital. Analysis of dispensing of long-acting IM antipsychotics: number of patients treated by olanzapine, risperidone and haloperidol. Analysis of $\mathrm{OP}$ prescriptions: number of patients, dosage and dose adjustment, treatment duration. Analysis of clinical data: diagnosis, treatment initiation and disruption, post-injection monitoring (blood pressure, heart rate, conscious state) and safety (other adverse events).

Results During the study period, 511 patients were treated by long-acting IM antipsychotics: $43 \%$ by haloperidol, $53 \%$ by risperidone and $4 \%$ by OP. OP was administered to 19 schizophrenic patients, mainly not compliant. In accordance with recommendations, a monthly dose of $405 \mathrm{mg}$ was prescribed initially for 4 patients, $300 \mathrm{mg}$ per 2 weeks for 1 patient, maintenance dosage after 2 months for 7 patients. 4 patients had only 1 injection. 3 patients required doses adjustments. 9 treatment disruptions were recorded during the study period for several reasons: care disruption, lost to follow-up, fear of injections. For the 10 patients currently treated, average treatment duration is 8 months. Post-injection monitoring data are collected on a special report form. Monitoring is performed for all injections in clinical departments. Altered consciousness has been reported in 1 patient during the 3 hours postinjection period without blood pressure or heart rate abnormalities and with normal vigilance 3 hours later. This suspected postinjection syndrome was notified to the pharmacovigilance services. Apart from this event, OP has been well tolerated.

Conclusions OP prescription is less frequent relative to other longacting IM antipsychotics, probably because of its recent availability, physicians' reluctance due to the risk of post-injection syndrome and requirement for hospitalisation and monitoring in the psychiatric department. This monitoring is strictly observed and reported in our hospital using our special form. Only one mild adverse effect was reported but confirms the importance of post-injection monitoring and continuing follow-up. $\mathrm{OP}$ is an additional therapeutic option for schizophrenic patients with poor compliance.

No conflict of interest.

\section{CPC-061 GLOBAL ANTIMICROBIAL STEWARDSHIP SURVEY - ANALYSIS OF UK RESULTS}

doi:10.1136/ejhpharm-2013-000276.518

1P Howard, ${ }^{2} \mathrm{C}$ Pulcini, ${ }^{3} \mathrm{D}$ Nathwani. 'Leeds Teaching Hospitals NHS Trust, Pharmacy, Leeds, UK; 'Université Nice-Sophia-Antipolis, Faculté de Médecine, Nice, France, ${ }^{3}$ University of Dundee, School of Medicine, Dundee, UK

Background Antimicrobial Stewardship (AMS) has been in existence since the early 1990s.

Purpose To measure the extent and components of global efforts in AMS.
Materials and Methods A 43-question survey was developed and tested using robust survey methodology, then refined - piloting in 11 countries across 6 continents - and disseminated worldwide.

Results Responses from the UK: 109 England, 10 Scotland, 9 Wales \& 3 Northern Ireland. Within the UK, 101 (79\%) have an Antimicrobial Stewardship Programme (ASP). The main barriers are lack of information technology and lack of personnel. In the $22(17 \%)$ that plan to develop an ASP the main barrier is lack of funding. Main ASP objectives were to reduce healthcare-acquired infection (91\%), improve outcomes $(57 \%)$, resistance $(47 \%)$ and reduce prescribing (46\%). $70 \%$ have an AMS policy, 92\% a formulary, $88 \%$ specific treatment and $83 \%$ prophylaxis guidance for all areas. AMS rounds exist in $86 \%$, resulting in reductions of antimicrobial (ATM) use in $36 \%$, increases in $14 \%$ and no change in $50 \%$.

Restriction of some ATMs occurs in $92 \%$ of hospitals: $84 \%$ restrict carbapenems, $88 \%$ quinolones, $91 \%$ cephalosporins. In $64 \%$ the pharmacy follows up. $12 \%$ practise diversity of ATMs and $5 \%$ cycle ATMs. $92 \%$ of ASPs report antimicrobial usage; 31\% link these data to resistance rates and $33 \%$ to infection rates. Only $6 \%$ have electronic prescribing for all patients.

The intranet is the most common communication method, followed by credit card, booklet, poster then smartphone app. All educate staff, mainly by with face to face induction followed by written information.

Of the 33\% who have formally reviewed their ASP, 100\% (15) showed reduction in inappropriate prescribing, 76\% (19) in broad spectrum antibiotics use, 71\% (15) in expenditure, 91\% (21) in healthcare-associated infections, $50 \%$ (3) in length of stay \& $54 \%$ (7) in resistance.

Conclusions Despite inherent limitations (e.g. response bias, unselected institutions, etc.), this survey suggests AMS can reduce antimicrobial resistance and expenditure, and should encourage a strategy to promote worldwide ASPs.

No conflict of interest.

\section{CPC-062 HEPATITIS C AND ADHERENCE}

doi:10.1136/ejhpharm-2013-000276.519

B Benítez García, L González del Valle, E Capilla Santamaría, T Perez Robles, A Herrero Ambrosio. Hospital Universitario La Paz, Pharmacy, Madrid, Spain

Background Good adherence to hepatitis C treatment seems necessary to obtain a successful treatment, increasing sustained virological response (SVR) rates.

Purpose To assess the adherence to chronic hepatitis $C$ treatment. Materials and Methods The study was descriptive, retrospective and observational. Patients with chronic hepatitis C, who were being treated with peginterferon and ribavirin or monotherapy with peginterferon in 2011, were selected. Data collected were: age, drug dispensed, duration of treatment, pretreatment, co-infected status (HIV, HBV), haemophilia status, genotype and viral load at the beginning and the end of treatment. Adherence was calculated taking into account the number of medicines dispensed and the dates.

Results Of the 113 patients included (102 adults, 11 children) 110 patients were treated with ribavirin and peginterferon. The other three patients were treated with only peginterferon. There were 32 patients with HIV co-infection and three haemophiliacs. The average adherence of 112 of patients was $103 \%$; one patient had less than $85 \%$ adherence. The genotype 1 patients $(n=54)$ had a mean duration treatment time of 35.5 weeks and a mean adherence of $103 \%$. The genotype non- 1 patients $(n=59)$ had a mean duration of treatment of 28.3 weeks and $104 \%$ adherence. The SVR of patients with genotype 1 and non- 1 were $50 \%$ and $60 \%$ respectively.

Conclusions There was a high rate of adherence to treatment because it has a definite time course. Adherence was greater than 
$100 \%$ owing to some patients coming to pick up the medicines before the set date. The method used in this study could be improved with validated adherence questionnaires. Good adherence is necessary to achieve SVR and it is especially important with the new protease inhibitors drugs (boceprevir and telaprevir), due to the complexity of triple therapy, adverse reactions and the high cost. Therefore, hospital pharmacists should collaborate on it with pharmaceutical care clinics specialising in hepatitis C.

No conflict of interest.

\section{CPC-063 HOW DO PHARMACISTS DOCUMENT AND TRANSMIT THEIR INTERVENTIONS? A SURVEY IN SEVERAL FRENCH-SPEAKING COUNTRIES}

doi:10.1136/ejhpharm-2013-000276.520

'S Kabiche, 'C Fonzo-Christe, ${ }^{2} \mathrm{~S}$ Prot-Labarthe, 'B Guignard, 'T Sigrist, ${ }^{2} \mathrm{~F}$ Brion, ${ }^{2} 0$ Bourdon, 'P Bonnabry. 'Geneva University Hospital, Pharmacy, Geneva, Switzerland; ${ }^{2}$ Robert Debré Hospital, Pharmacy, Paris, France

Background The role of a clinical pharmacist in providing and transmitting drug information to other health professionals varies greatly between countries. There is no consensus on the most efficient way to document and transmit interventions and its effect on the implementation of recommendations in practise.

Purpose To describe and then compare the methodology of pharmacist's interventions (PIs) in each of the following French-speaking countries: France, Switzerland, Belgium and Quebec.

Materials and Methods 527 on-line questionnaires were distributed (276 in France, 47 in Switzerland, 92 in Belgium, and 112 in Quebec). They contained 36 questions about clinical pharmacy work, the ways of transmitting information and its documentation in the patient record.

Results 160 hospitals answered (total 30.3\%; France 33.7\%, Switzerland 44.7\%, Belgium 23.9\%, Quebec 21.4\%). In the Swiss hospitals, only $47.4 \%$ of pharmacists analysed pharmaceutical prescriptions while $97.4 \%$ did in France, $76.5 \%$ in Belgium and $100 \%$ in Quebec. The same trend could be seen while examining the pharmacist's presence on the wards: $42.1 \%$ in Switzerland, $58.4 \%$ in France, $85.7 \%$ in Belgium and $88.2 \%$ in Quebec.

Communications channels for PIs also differed depending on countries: Swiss pharmacists mainly used the phone $(56.7 \%$ of the cases), followed by personal visits (30.7\%). In France and Quebec the preferred methods were writing notes in the patient's record in respectively $39.1 \%$ and $36.4 \%$ of the cases, followed by phone calls in $25.4 \%$ and $32.4 \%$. In Belgium, the communication of PIs was most frequently done through personal visits (40\%).

Conclusions Pharmacist's interventions in terms of ways of transmitting drug information and its documentation differ among the 4 countries. Differences in the pharmacist's integration into the ward teams, access to the patient record file and to the medical prescription probably explain the heterogeneity of our results.

No conflict of interest.

\section{CPC-064 HOW IS IT BEST TO REPORT PHARMACEUTICAL INTERVENTIONS TO A MEDICAL TEAM? A CLINICAL RELEVANCE ASSESSMENT}

doi:10.1136/ejhpharm-2013-000276.521

${ }^{1} \underline{C}$ Di Fiore-Fave, 'E Bouvet, 'M Vié, ${ }^{2} \mathrm{P}$ Massip, ' $\mathrm{IM}$ Canonge. ${ }^{1} T o u l o u s e$ University Hospital, Clinical Pharmacy, Toulouse, France; ${ }^{2}$ Toulouse University Hospital, Infectious and tropical diseases medical department, Toulouse, France

Background The clinical pharmacy department has recently started working with the medical team of the infectious and tropical diseases department. A pharmacy student, supervised by a clinical pharmacist, cheques 28 patient prescriptions daily.
Purpose To evaluate the impact and quality of pharmaceutical interventions (PIs) issued over a period of 8 months.

Materials and Methods All interventions are recorded and coded according to the criteria defined by the working group of the French Society of Clinical Pharmacy [1]. A note of the relevance is attributed by the pharmacist to each PI, according to Bayliff and Einarson's scale [2].

Results In total, 1947 paper prescriptions were analysed. During this period, 980 patients were hospitalised, $133(13.6 \%)$ were identified as having 209 PIs. Physicians accepted 168 interventions (80\%), of which the pharmacist quantified the clinical relevance. A very significant clinical impact (level 2) was attributed to 36 PIs (21.5\%), a significant clinical impact (level 1) to 77 (46\%) and 54 PIs (32.5\%) had an informative objective (level 0). No interventions had a vital clinical impact (level 3).

For each level of relevance, the distribution of PIs was described according to the type of drug-related problems on the one hand and the type of pharmacists' recommendations on the other hand Highlighting the clinical impact of PIs increased the interest of physicians in pharmaceutical work. Consequently, they asked for pharmaceutical reports more frequently (twice a month instead of once a year).

Conclusions The results reinforce the idea that a regular presence in care encourages collaboration between pharmacists and health care teams.

\section{References}

1. Bedouch P, Charpiat B, Roubille R, et al, (2007). Site internet de la société française de pharmacie Clinique pour l'analyse des interventions pharmaceutiques: finalités, mode d'emploi et perspectives. J Pharm Clin 26(1), 40-4.

2. Bayliff CD, Einarson TR, (1990). Physician assessment of pharmacist's intervention: a method of estimating cost avoidance and determining quality assurance. Can J Hospi Pharm 43(4), 167-7.

No conflict of interest.

\section{CPC-065 HOW TO ASSESS MEDICATION ADHERENCE AMONG PATIENTS WITH RESISTANT HYPERTENSION TREATED WITH TWO DIFFERENT PHARMACOLOGICAL INTENSIFICATION STRATEGIES}

doi:10.1136/ejhpharm-2013-000276.522

${ }^{1} \mathrm{H}$ Beaussier, ${ }^{2} \mathrm{~F}$ Coudore, ${ }^{3} \mathrm{G}$ Bobrie, ${ }^{4} \mathrm{~S}$ Peyrard, ${ }^{5} \mathrm{~L}$ Tran, ${ }^{5} \mathrm{Y}$ Bezie, ${ }^{6} \mathrm{M}$ Azizi, ${ }^{2} \mathrm{P}$ Boutouyrie. ${ }^{1}$ Groupe Hospitalier Paris Saint-Joseph, CRC Pharmacie, Paris Cedex 14, France; ${ }^{2} \mathrm{HEGP}$ Service de Pharmacologie Clinique, Paris Cedex 15, France; ${ }^{3} H E G P$, Service de médecine vasculaire - Unité d'hypertension artérielle, Paris Cedex 15, France; ${ }^{4} H E G P$, URC, Paris Cedex 15, France; ${ }^{5}$ Groupe Hospitalier Paris Saint-Joseph, Service de Pharmacie, Paris Cedex 14, France; ${ }^{6} \mathrm{HEGP}$, CIC, Paris Cedex 15, France

Background Non-adherence to medicines and lifestyle are the main contributors to resistance to antihypertensive treatment (AHT). Various measures to assess medicines adherence (MA) among patients with resistant hypertension (RH) have been proposed but none is fully effective.

Purpose To assess MA with a new scoring system in RH patients included in a randomised controlled trial and the characteristics associated with low MA

Materials and Methods Patients with RH on 4 week-treatment with irbesartan $300 \mathrm{mg}+$ hydrochlorothiazide $12.5 \mathrm{mg}+$ amlodipine $5 \mathrm{mg}$, were randomised to either reinforcement of sodium depletion by sequential administration of spironolactone and other diuretics ( $A B$ group, $n=82$ ) or reinforcement of renin angiotensin system blockade by sequential administration of ramipril 5-10 mg and bisoprolol 5-10 mg ( $\mathrm{RB}$ group, $\mathrm{n}=82$ ) for 12 weeks. In accordance with the literature, 4 methods were used to evaluate MA: 1/ measurement of plasma irbesartan concentration (HPLC); $2 /$ measurement of urinary AcSDKP/creatinine ratio (UR) to evaluate ACE inhibitor exposure; $3 /$ last dose of medicine taken before visit; 4/pill 